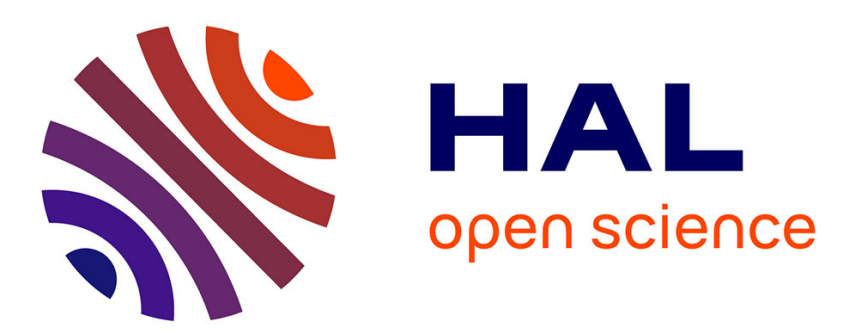

\title{
Integrating and sequencing flows in terminal maneuvering area by evolutionary algorithms
}

Catya Zuniga, Daniel Delahaye, Miquel Angel Piera

\section{To cite this version:}

Catya Zuniga, Daniel Delahaye, Miquel Angel Piera. Integrating and sequencing flows in terminal maneuvering area by evolutionary algorithms. DASC 2011, 30th IEEE/AIAA Digital Avionics Systems Conference, Oct 2011, Seattle, United States. pp 1-32; ISBN : 978-1-61284-797-9, 10.1109/DASC.2011.6096177 . hal-00912819

\section{HAL Id: hal-00912819 \\ https://hal-enac.archives-ouvertes.fr/hal-00912819}

Submitted on 27 Jan 2014

HAL is a multi-disciplinary open access archive for the deposit and dissemination of scientific research documents, whether they are published or not. The documents may come from teaching and research institutions in France or abroad, or from public or private research centers.
L'archive ouverte pluridisciplinaire HAL, est destinée au dépôt et à la diffusion de documents scientifiques de niveau recherche, publiés ou non, émanant des établissements d'enseignement et de recherche français ou étrangers, des laboratoires publics ou privés. 


\title{
Integrating and Sequencing Flows in Terminal Maneuvering Area by Evolutionary Algorithms
}

\author{
Catya Zuñiga UAB, Edifici Q Depto. TESA 08193 Bellaterra, Spain (CatyaAtziry.Zuniga@uab.cat) \\ Daniel Delahaye ENAC 7, Ave Ed Belin 31055 Toulouse France (delahaye@recherche.enac.fr) \\ Miquel A. Piera UAB, Edifici Q Depto. TESA 08193 Bellaterra, Spain (MiquelAngel.Piera@uab.cat)
}

\begin{abstract}
This paper presents a new approach for merging multiple aircraft flows in a Terminal Maneuvering Area (TMA). This work is motivated by the current overloaded airspace near large airports and the need of more efficient methods to help controllers. Some attempts to alleviate airspace congestion such as the minimum spacing requirements, negotiation of voluntary reductions in scheduled service, and the construction of additional runways at major airports have been done. Even though, more fundamental changes are needed to improve the use of available air capacity. Present research consists of a new approach to optimize a set of aircraft planned to land at a given airport; it is proposed to merge the incoming flows from different routes by mean of speed and path changes. Due to the high combinatoric induced by such a problem, a stochastic optimization algorithm has been developed in order to propose to each aircraft a new route and speed profile. Those changes aim to remove conflicts at merging points and to maintain separation of aircraft following the same route link according to their wake turbulence constraint. The optimization criteria is based on the minimum deviation from the initial path planning. This algorithm has been successfully applied to Gran Canaria airport in Spain with real traffic demand samples for which conflict free flow merging is produced smoothly with optimal runway feeding.
\end{abstract}

\section{Introduction}

"Some aspects of the Air Traffic Management

remain cast in stone for decades, whilst other change more rapidly than the time it takes to write a book about them" [7].
Future demand in Air Traffic Management (ATM) systems is expected to increase at an average yearly rate of 2.2 to 3.5 in the long term period up to 2030 [12]. This future situation will generate airspace sectors that operate at or above its current capacity, hence different ATM modernization projects have been started. The Single European Sky ATM Research (SESAR) launched by the European Community and the Next Generation Air Transportation System (NextGen) which launched by US government are future projects aim to ensure the safety and fluidity of air transport over the next thirty years.

These concepts address all services related to air navigation specially Air Traffic Flow Management (ATFM) which supports the use of available airspace effectively, including airport capacity and therefore, its importance has been increased significantly. Hence, major benefits can be expected if areas with a high traffic density like the Terminal Maneuvering Area are analyzed to assess the performance of new ATM concepts, like 4D-trajectory planning and strategic deconfliction allowing ATC efficient procedures to predict conflicts among trajectories. As one of the bottlenecks impeding ATM performances is the merging arrival operations into the TMA. This work is mainly focused on optimizing the number of conflicts to improve capacity into the TMA sector.

The Terminal Maneuvering Area or Terminal Control Area (TMA) is a block of airspace class around airport; it is a special type of airspace designed to handle aircraft arriving to and departing from airports and perhaps one of the most complex types of airspace. Current TMA are being forced to accept more and more flights each day, and departure pushes to accommodate late arriving flights; as a consequence, 
delay flight are induced causing further up \& downline disruptions. This, plus the inherent random nature of the arrival times are some of the reasons what make TMA as one of the main bottleneck on ATM. These random arrivals, must be converted into an orderly stream while merging aircraft flows coming from different entry points, commonly done in a short time horizon (about 45 minutes) [1].

Therefore, there is a need for tools that allow an efficient TMA which minimize the deviation between desired and conflict free trajectory for each aircraft while merging multiple flows into the TMA and delivering precisely spaced to the runway threshold. Predictable arrival flows where aircraft land at a specified time, enables ground controllers to issue a more efficient TMA flow system. Moreover, it provides shorter and safetier flights using more efficient airspace by increasing airspace capacity [15].

\section{Overview}

The arrival phase of flights, typically starts when the aircraft leave their cruise level in En-Route phase and ends when aircraft reach the Final Approach Fix (FAF) as depicted in Figure 1. Aircraft arriving to airports in terminal areas are organized in arrival streams. To build such streams, the individual paths of each aircraft have to be gradually merged. An aircraft approaching is still mainly controlled by ATC with means of radar vectoring where ATC sends additional instructions to aircraft, depending on the actual traffic, to avoid dangerous encounters. Finally, a sequence and merge procedure is made to position aircraft into a single stream (or multiple streams if multiple runways are being used). They must recognize and select the proper technique by which they can alter an aircraft's position within a flow or, the spacing between the leading and the following aircraft. Although this method is efficient and flexible, it is highly demanding for air and ground sides under high traffic load conditions, as it imposes rapid decisions for the controller and time-critical execution by the flight crew.

With the introduction of more Area Navigation (RNAV) arrival and departure procedures, there are potential benefits to be achieved such as; reducing the need to vector aircraft; reduced voice communication; improved situational awareness; reduced flying time

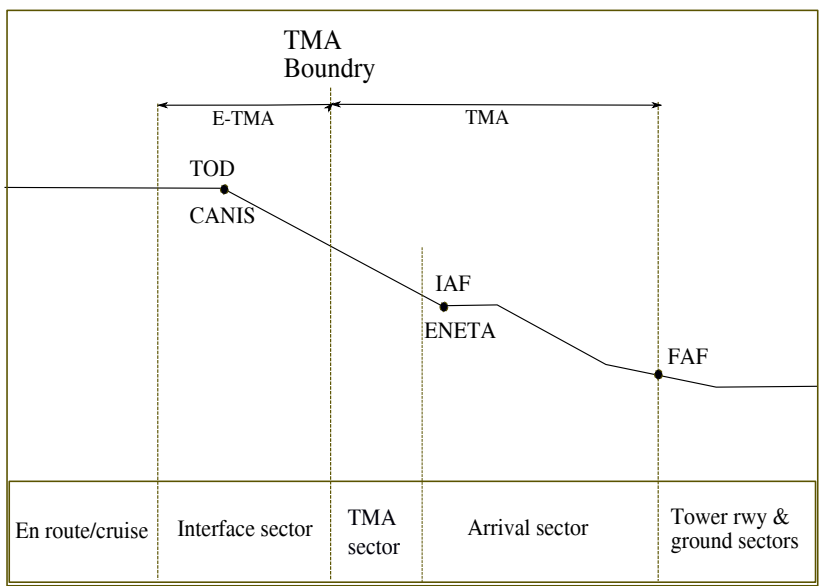

Fig. 1. Control phases and sectors for the arrival phase of flight

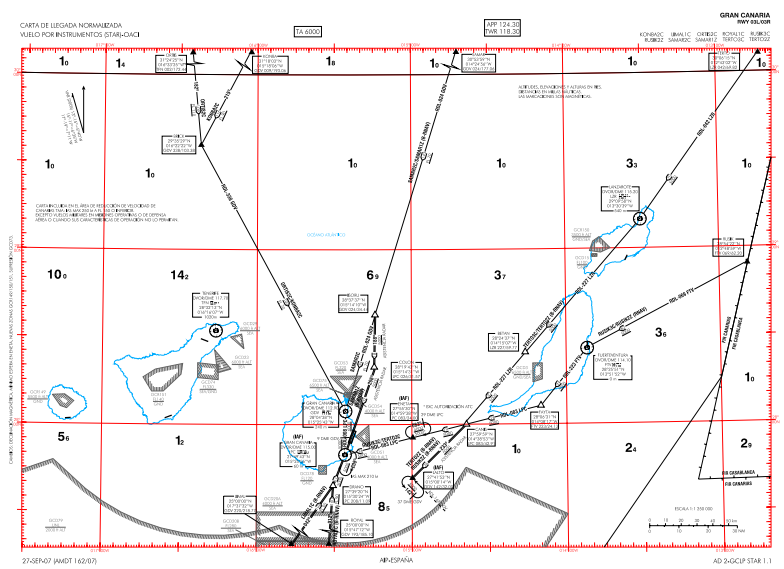

Fig. 2. Gran Canaria STAR

and distance; and, improved predictability. RNAV procedures refer to the ability to execute point to point navigation. Standard Instrument Departures routes (SIDs) and Standard Terminal Arrival Route (STARs) are both very similar in many aspects e.g. offering the pilot pre-planned Instrumental Flight Rule (IFR) procedures. STARs are designed to expedite ATC arrival procedure and facilitate the transition between en-route and instrument approach segment as well as to streamline approach flows and to give a more regular approach to an airport. Incoming flows are progressively merged into a single flow for each active landing runway. As example of a STAR, Figure 2 presents Gran Canaria TMA.

The introduction of RNAV at airports allows operations, based on a common set of design and opera- 
tional principles, to ensure consistent levels of flight safety. The enhanced predictability and repeatability of RNAV procedures leads to efficiency and environmental benefits being afforded to both airspace users and air navigation service providers.[10]. These procedures should rely on appropriated navigation aids such as instrument assisted landing systems and satellites. The development and application of the Global Positioning System (GPS) and the Automatic Dependant Surveillance Broadcast (ADS-B) allows aircraft to obtain highly accurate positional and directional information about them and other nearby aircraft.[15]

The objective of the algorithm is to effectively merge the arrival flows while keeping aircraft on lateral navigation. This will allow to decrease ATC workload and tactical intervention while keeping a safety separation distance and a efficient landing sequence.

\section{Previous Related Works}

Researchers have been studying the problems related to ATM and diverse traffic control strategies have been proposed. Conflict Detection and Resolution (CD\&CR) algorithms have been designed to improve ATM performance. A search of the literature was conducted to identify concepts related to terminal merges and sequence problems as part of these traffic control strategies.

An overview of different CD\&CR algorithms is being provided in [17] where over 60 different methods have been analyzed and classified. Some of the methods presented are currently in use or under operational evaluation for example [13], the user interface of TCAS.

Some of these methods include tactical approaches focusing on resolving immediate conflicts, with limited consideration of future postconflict routing and planning. Most of the methods are designed for enroute sectors and based on Optimization approaches to treat the CD\&CR problem such as; Genetic Algorithms as in [20] where autonomous aircraft are enable to maneuver freely while maintaining separation assurance from traffic and area hazards; Other CR algorithm, which uses a combination of patternbased maneuvers and Genetic Algorithms to achieve resolution, is presented in [24]; The Ant Colony
Optimization (ACO) algorithm has also been applied to solve problems involving autonomous aircraft by applying the path planning problem, [8]; or The developed a mathematical model is formulated as a mixed integer linear program in [23] using concepts based on speed control and flight-level assignments for conflict resolution over predefined routes; other approach applying Linear Programming is [21] where a system of multiple aircraft is deconflicted by applying the path planning problem among given waypoints. Semi-definite Programming is utilized in [11] where each aircraft proposes its desired heading while a centralized air traffic control authority resolves any conflict arising between aircraft. The approaches mentioned above are some of the most interesting works and concepts to deal with the CD\&CR problem.

In [4] a broader suite of concepts have being investigated to address merging and spacing problems arising from structured RNAV and Required Navigation Performance (RNP) routes in the terminal environment referred to as Spacing of Performance-based Arrivals on Converging Routes (SPACR). It addresses the nearterm merging and spacing problem. A method to merge arrival flows of aircraft without using heading instructions is presented in [5], [18], [14], [10]. The principle is to achieve the aircraft sequence on a point with conventional direct-to instructions, using predefined legs at iso-distance to this point for path shortening or stretching.

Different approaches for the landing sequence problem have been also studied such as [3] based on Linear Programming which solves the static case presenting a mixed-integer zero-one formulation of the problem together with a population heuristic algorithm. A Dynamic-Programming-based approach which used a method called Constrained Position Shifting (CPS) as in [1] and [6] and [2] is another class of algorithms that is able to handle commonly-encountered operational constraints for the sequence problem.

The algorithm developed in this paper will aim to find the optimal trajectory and the optimal distance to be flown for a set of aircraft using Genetic Algorithms. A new combinatorial optimization algorithm to detect and solve conflicts into the TMA is presented. The algorithm is based on the premise of achieving an optimum system-wide improvement in performance instead of accurately and precisely space individual 
aircraft. It was also based on the idea that a following aircraft, on the same flight path as the lead, would maintain distance-based, rather than a time-based, spacing interval from the preceding aircraft.

The reminder of this works is as follows; the hypotheses needed to formulate the CD\&CR optimization problem, the assumptions made, and the Mathematical Modeling is given in the Problem modeling section. Conflict avoidance constraints are formulated as part of the resolution strategy. In the next section, Evolutionary Algorithms are introduced. and the Genetic Algorithm approach is presented in the section called Application to our problems. In the result section, numerical examples are introduced and solved from a sample simulation study of 35 and 50 aircraft. Conclusions are discussed at the end of the paper.

\section{Problem Modeling}

Three cases to avoid possible conflicts are considered in the work of [21]:

1) Aircraft is allow to change speed but trajectory remains fixed. This case is called the velocity change problem (VC problem);

2) Aircraft fly at the same speed profile and it is only allowed to change its trajectory. This case is called the heading angle change problem (HAC problem).

3) the mix of both HAC and VC problem.

The present approach belongs to the third category presented above; conflicts can be avoided by speed regulations or/and path changes. Merging and sequencing arrival flows requires the ability to speed up or delay aircraft, specially in dense traffic areas, speed regulations may not be sufficient and the use of an alternative trajectory may become necessary.

The system architecture proposed in this work consist of a route network compose by sub-routes in which aircraft are allowed to fly as illustrated in Figure 3. Each route has a defined number of subroutes designed to change path if necessary to solve conflicts. Sub-routes have a predefined length which depend on the length of the original route. Lateral deviation provides the controller and pilot with improved situational awareness. Sub-routes are properly separated laterally, and are composed of links; A link is defined as a portion of a route which

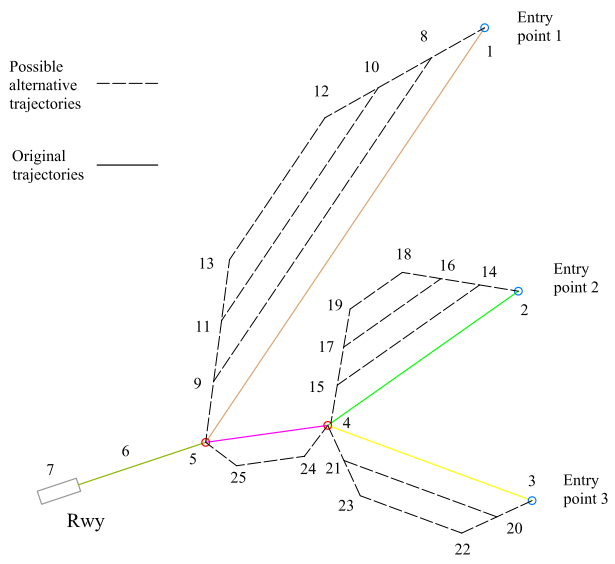

Fig. 3. TMA configuration example

connect two waypoints.

To illustrate these concepts, see Figure 3; A trajectory is formed from Entry point 3 (or Node No.3) to runway (or Node No.7) passing by Node No.4 and 5. For this original trajectory, 3 subroutes are defined between Node No.3 and Node No.4, and one sub-route from Node No.4 to Node No.5.

The core problem that is considered on this work is to find conflict free trajectories for a given set of aircraft landing at the same airport by changing their paths and (or) their speeds. Two kind of conflicts will be considered :

- Node Conflict : Minimum space separation has to be manage between successive aircraft flying over the same node.

- Link Conflict : aircraft flying on the same link has to be separated by a minimum separation distance depending on the aircraft's wake turbulence category.

When the separation between two planes is smaller that the minimum separation criteria then a Conflict is Detected (CD). The separation criteria (SC) is due to many factors; first of all, there are aerodynamic considerations; the risk of instability if an aircraft interacts with the wake-vortex turbulence of another aircraft landing or taking off before it; SC also depends on the class of the two aircraft (the leading and the trailing); another one depends on the density of air traffic and the region of the airspace, to mention some factors. 


\begin{tabular}{|l|ccc|}
\hline & heavy & medium & light \\
\hline heavy & 4 & 3 & 3 \\
medium & 5 & 3 & 3 \\
light & 6 & 4 & 3 \\
\hline
\end{tabular}

TABLE I

SEPARATION Minima [NM] ICAO DOC-4444

A largely accepted value for horizontal minimum safe separation between two aircraft at the same altitude is $5 \mathrm{nmi}$ in general enroute airspace; this is reduced to $3 \mathrm{nmi}$ in approach sectors for aircraft landing and departing. As different rules exist, separation standards are established by the International Civil Aviation Organization (ICAO). The nominal horizontal minimum spacing interval that will be considered in this work are given in Table I.

Some assumptions and simplifications were made in the modeling and execution of the experiment as in [9]. In the model, aircraft trajectories were entirely deterministic. Aircraft executed their planned trajectories and conflict resolution trajectories perfectly, no data-link transmission delays, nor pilot-action delays. Uncertainties in the estimation of aircraft position or unexpected wind fields are not consider. It should be pointed out, that in absence of wind the TAS speed can also be considered as Ground Speed. As such, trajectory conflicts could be predicted with perfect accuracy over any time horizon, and resolution trajectories could be assured to be conflict free. Once a resolution trajectory was determined by the automation, it was executed immediately and precisely. The aircraft are assumed to fly highest/lowest permissible speeds over each flight segment ( $\pm 20 \%$ of its average GS) and they are assumed to fly within a fixed altitude layer. The routes from different directions towards final approach are adequately separated to avoid merging of traffic; and lastly, arrival traffic management initiatives such as miles in trail restrictions, time-based metering, or ground-delay programs were not modeled.

\section{Mathematical Modeling}

The TMA has been modeled by a graph $G=$ $\{\mathcal{N}, \mathcal{A}\}$ as shown on Figure 3 for which $\mathcal{N}$ is the set of nodes and $\mathcal{A}$ is the set of links. This graph gathers together the original links (links $\{(1,5) ;(2,4) ;(4,5) ;(3,4) ;(5,7)\}$ on Figure 3$)$ and the sub-routes links for alternative options (for instance link $(4,5)$ on Figure 3 may be substituted by the subroute $\{(4,24),(24,25),(25,5)\})$. For each link $l_{i}$ the set of alternatives is noted as alt $\left(l_{i}\right)$.

Let $\mathcal{F}$ be the set of flights planned to land in a given time horizon $\left[0, T_{\max }\right]$. For each flight $f_{i}$ in $\mathcal{F}$ we know the following :

- $e_{i}$ : entry point of flight $f_{i}$ in the TMA. Such entry points are nodes in the graph $(\{1,2,3\}$ in our case) ;

- $t_{i}$ : time of flight $f_{i}$ at entry point.

- $v_{i}$ : speed of aircraft $\left(f_{i}\right)$

- $w t_{i}$ : wake turbulence category (heavy, medium, light)

For a given route $r_{j}$ flown by an aircraft we will consider the associate set of alternatives according the links belonging to such route. We will note such alternatives by : Alt $\left(r_{j}\right)=\prod_{k=1}^{k=L\left(r_{j}\right)}$ alt $\left(l_{k}\right)$. Where $L\left(r_{j}\right)$ is the number of links of route $r_{j}$ having alternatives choices. For each aircraft involved in the simulation one has to choose optimal route and speed in order to avoid conflicts and to insure wake turbulence separation in the terminal area.

Depending on the route used, aircraft may have more or less options for the path planning. For instance (see Figure 3), an aircraft entering entry point 1 has alternative choices only on link $(1,5)$ but an aircraft entering entry point 2 has alternative choices on links $(2,4)$ and $(4,5)$. So our state space $X$ may be summarized by the following table :

$$
\vec{X}=\begin{array}{|l|l|l|l|}
\hline \vec{a}_{1} & \vec{a}_{2} & \ldots & \vec{a}_{|\mathcal{F}|} \\
\hline v_{1} & v_{2} & \ldots & v_{\mid \mathcal{F}} \\
\hline
\end{array}
$$

where $\vec{a}_{i}$ is the vector of alternatives of flight $f_{i}$ and $v_{i}$ is the associated speed. It must be noted that dimensions of vectors $\vec{a}_{i}$ may differ according to the routes used by the aircraft.

We are searching a point in this state space which minimizes the total number of conflicts :

1) Wake turbulence "conflict" : $\forall f_{i}, f_{j} \in \mathcal{F}$ flying on the same link and for which $f_{i}$ is the leader, $d\left(f_{i}, f_{j}\right)>s_{i j} \forall t \in[0, T]$, where $d\left(f_{i}, f_{j}\right)$ is the distance separating aircraft $f_{i}, f_{j}, s_{i j}$ is the separation standard which depends on the aircraft's wake turbulence category. 
2) Conflicts on node : when an aircraft $f_{i}$ is flying over a node $n_{k}$, other aircraft have to be $5 \mathrm{NM}$ away from the node.

This optimization process is subject to the following constraint :

- Speed constraint : $\forall f_{i} \in \mathcal{F} v_{i} \in\left[v_{i_{\min }}, v_{i_{\max }}\right]$

Having discrete and continuous decision variables such problem belongs to the class of mix optimization problems.

The combinatoric associated to the discrete part can be summarized by the following formula :

$$
|X|=\prod_{i=1}^{i=|\mathcal{F}|} \prod_{k=1}^{k=L\left(r_{i}\right)} \operatorname{alt}\left(l_{k}\right)
$$

For instance, for 50 aircraft with an average number of alternatives equal to 5 , the induce combinatoric is about $5^{50}$. Furthermore, the objective function is non linear, not convex and not separable. The state space is not connected meaning that deterministic optimization approach are not suitable for such problem. This problem is NP_Hard. We have then developed a stochastic approach based on evolutionary algorithms which are detailed in the next section.

\section{Evolutionary Algorithms}

Evolutionary algorithms use techniques inspired by evolutionary biology such as inheritance, mutation, natural selection, and recombination (or crossover) to find approximate solutions to optimization problems [12], [19], [16], [22]. An individual, or solution to the problem to be solved, is represented by a list of parameters, called chromosome or genome. Initially several such individuals are randomly generated to form the first initial population (POP $(\mathrm{k})$ on Figure 4).

Then each individual is evaluated, and a value of fitness is returned by a fitness function. This initial population undergo a selection process which identify the most adapted individual. The one which has been used in our experiments is a deterministic $(\lambda, \mu)$-tournament selection. This selection begins by randomly selecting $\lambda$ individuals from the current population $(\mathrm{POP}(\mathrm{k})$ and keeps the $\mu$ bests $((\lambda>\mu))$. This two steps are repeated until a new intermediate population $\left(\mathrm{POP}_{i}\right)$ is completed. Following selection, one of the three following operators is applied : nothing, crossover, and mutation. The associated probability of application are respectively $\left.\left(1-p_{c}-p_{m}\right)\right), p_{c}$ and $p_{m}$. Crossover results in

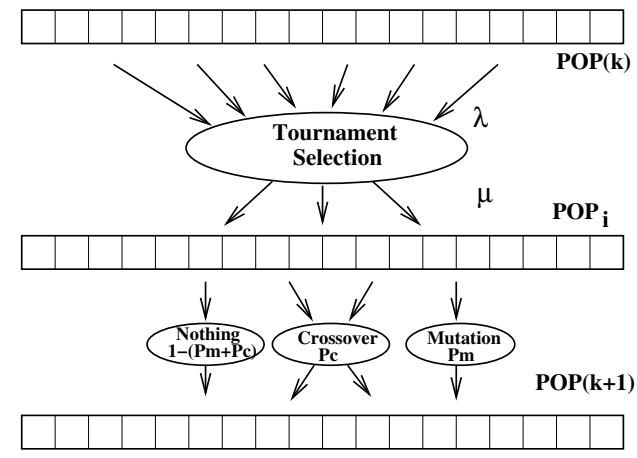

Fig. 4. Genetic Algorithm with Tournament Selection. The first step consists in the selection of the best individuals from population $P O P(k)$. Afterward, recombination operators are applied in order to produce the $P O P(k+1)$ population.

two new child chromosomes, which are added to the next generation population. The chromosomes of the parents are mixed during crossover. These processes ultimately result in the next generation population of chromosomes (POP $(k+1)$ on Figure 4) that is different from the initial generation. This generational process is repeated until a termination condition has been reached. The next section presents the application of EA to our problem.

\section{Application to our problems}

\section{Coding}

In order to make run the GA on such problem, one first has to design an efficient coding easily managed by recombination operators. We fist consider the set of aircraft involved in a given time window for which one want to optimize the merging and sequencing for landing. For each aircraft, one has to find an optimal route and some speed regulations. The coding is then summerized by a table which gather together all the dcisions variable for all the aircraft involved in the time window (see Figure 5). The chromosome consists in two part. The first part is link to the speed changes (blue) and the second one decribes the alternatives route for a given aircraft. Depending of the entry point, aircraft may have different number of alternative. For instance, aircraft entering TMA (see Figure 3) by the first entry point have only one alternative but the one entering by the others point (2 and 3 ) have two. In order to memorize the 


\section{Aircraft List}

\begin{tabular}{|l|l|l|l|l|l|l|l|}
\hline$\delta_{1}$ & & $\delta_{\mathbf{i}}$ & & & & & $\delta_{\mathbf{N}}$ \\
\hline $\mathbf{A}_{11}$ & & $\mathbf{A}_{\mathrm{i} 1}$ & & & & & $\mathbf{A}_{\mathrm{N} 1}$ \\
\hline $\mathbf{A}_{12}$ & & $\mathbf{A}_{\mathrm{i} 2}$ & & & & \\
\hline $\mathbf{n}_{1}$ & & $\mathbf{n}_{\mathrm{i}}$ & & & & & $\mathbf{n}_{\mathbf{N}}$ \\
\hline
\end{tabular}

Speed Changes

Route Decisions

A Posteriori

Conflict number

Fig. 5. State Space coding

performances of a given decision, we put an extra table which gather the number of conflicts a given aircraft has encontered on his route. This information will be used by the recombination operators in order to focus on aircarft involved in conflict.

\section{Initialization}

In order to make run the Evolutionary process, one must be able to initiate a population of chromosomes. If no information about the structure of optimal solutions is available at the beginning of the optimization process, one can use a uniform random trial to initialize the population.

\section{Crossover}

The crossover operator is a bias uniform crossover for which each gene is check in order to be put in the next generation. For each gene $i$ (aircraft), we compute the sumation $S$ of conflict number in both parent $P_{1}$ and $P_{2}: S=n i\left(P_{1}\right)+n i\left(P_{2}\right)$. Then, we compute the probability $P_{C}$ to transfer the decision variable of gene $i$ in both children :

$$
P_{c}=1.0-\frac{n i\left(P_{1}\right)}{S}
$$

When there is no conflict in both parent such probability is set to $\frac{1}{2}$. Based on a uniform random trial between 0 and 1 , we decide when gene from parent $P_{1}$ or parent $P_{2}$ is inserted into children chromosomes (see Figure 6).

\section{Mutation}

As for the crossover operator, a bias is introduced in order to focus on aircraft involved in conflict. To do that we beging to compute the total number of conflict

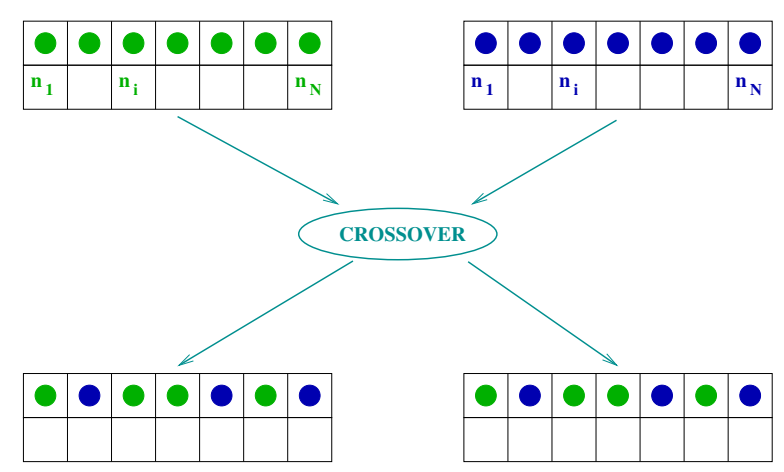

Fig. 6. Bias Uniform Crossover.

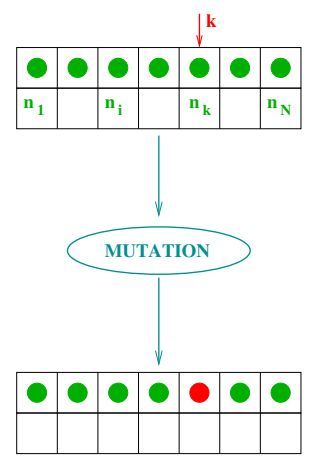

Fig. 7. Bias Mutation.

in the chromosome :

$$
N_{\text {conf }}=\sum_{i=1}^{i=N} n_{i}
$$

Then we generate a random number $p$ between 0 and 1 (uniform distribution) and we compute the cumulative following summation :

$$
S(k)=\sum_{i=1}^{i=k} \frac{n_{i}}{N_{\text {conf }}}
$$

till $S(k)<p$ for which the gene $k$ is then mutated (see Figure 7). Depending of the configation of the GA, the mutation may change the speed of an aircraft, its route or both.

\section{Fitness Computation}

The fitness computation is based on a simulation of the traffic in the TMA based on the decision variables of a given individual. For each aircraft, we compute its track on the route network (nodes and links). Such track consists of a set of positions 
of the aircraft every 10 seconds. Those tracks are then used to compute the conflict appearing on nodes and links. This first computation represents the first objectives we have to set to zero $\left(y_{1}\right)$. Then, for a given planning, the speed changes and extra distance are computed for all aircraft in order to build the second objective $y_{2}$. Finally, we gather together those two previous objectives into a single fitness that has to be maximized :

$$
\text { fitness }=\frac{1}{0.01+y_{1}}+\frac{1}{0.01+y_{2}}
$$

This fitness reach 200 value when both objectives $y_{1}, y_{2}$ are equal to zero.

Based on the results of such simulation, it is possible to update the conflict number for each aircraft in order to guide recombination operators.

\section{Results}

The algorithm has been tested on the Gran Canaria terminal maneuvering area (see Figure 2). This associated model is given on Figure 3 for which four link have been extended with some alternative routes. Two scenarios have been investigated with 35 and 50 aircraft respectively on the same time period of 1 hour.

\section{Results for the first scenario}

\section{Scenario Description}

A synthetic STAR configuration of the Gran Canaria TMA has been used (as depicted in Fig. 8 ) to test the benefits of the proposed algorithm. In the arrival phase, three routes fuse into one single route towards the final approach (runway 03L/03R) by merging in two different waypoints. Three different routes were defined, two correspond to current STARs defined in the Spanish Aeronautical Information Publication (AIP), TERTO3C and RUSIK3C, and one additional STAR was defined in order to complicate the traffic flow in the scenario presented. This last STAR is defined by the name NPWT3C. The waypoints sequence for each of the three STARs is as follows :

- TERTO3C: TERTO, LZR, BETAN, CANIS, ENETA (IAF), LPC (FAF), RWY

- RUSIK3C: RUSIK, FTV, FAYTA, CANIS, ENETA (IAF), LPC (FAF), RWY

- NWPT3C: NWPT, FAYTA, CANIS, ENETA (IAF), LPC (FAF), RWY

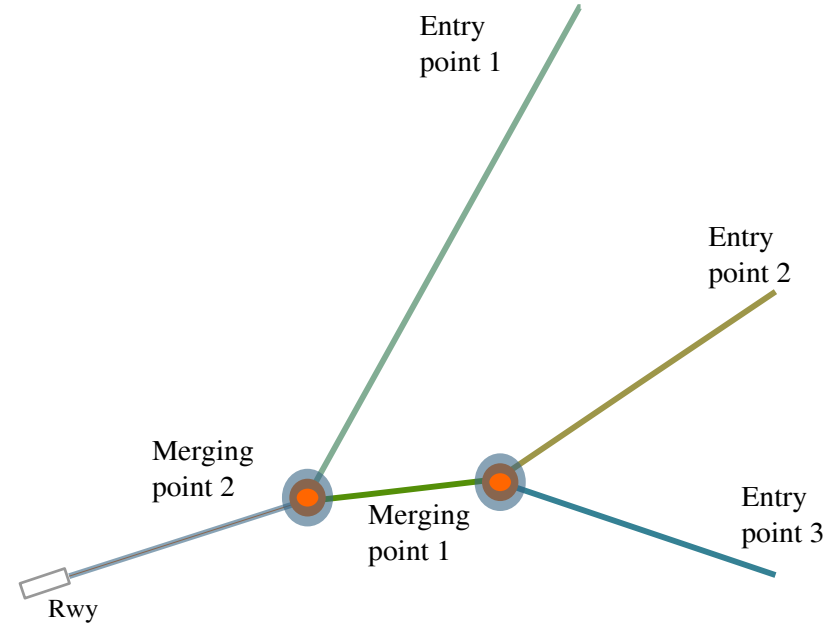

Fig. 8. Synthetic Gran Canaria STAR

The algorithm is designed to solve detected conflicts in an horizon between TMA entry point to the initial approach fix (IAF) point by amending either the speed or the trajectory of the aircraft such that the conflict is solved, no new or secondary conflicts are produced, and the aircraft accomplish its original required arrival time.

The GA parameters used for the first scenario are the following :

\begin{tabular}{|c|c|}
\hline Pop size & 100 \\
Number of generation & 20 \\
Probability of Crossover & 0.3 \\
Probability of Mutation & 0.3 \\
\hline
\end{tabular}

The Figure 9 show the evolution of the fitness features with generation.

The experiment has been done on a $3 \mathrm{Ghz}$ Pc based on a Java code. The evolution of the fitness features are summarized on Figure 9 for which the fitness of the best individual, the average fitness on population and the standard deviation are plot with generations. As it can be seen on Figure 9 the EA (Evolution Algorithm) find an optimal solution $\left(y_{1}=y_{2}=0\right.$, fit $\left.=200\right)$ solution after 42 generations.

The associated evolution of criteria is given on Figure 10. The conflicts on links are first eliminated after 10 generation and conflicts on nodes disappear after generation 42 .

\section{Results for the second scenario}

Having more aircraft in this scenario, the EA pa- 


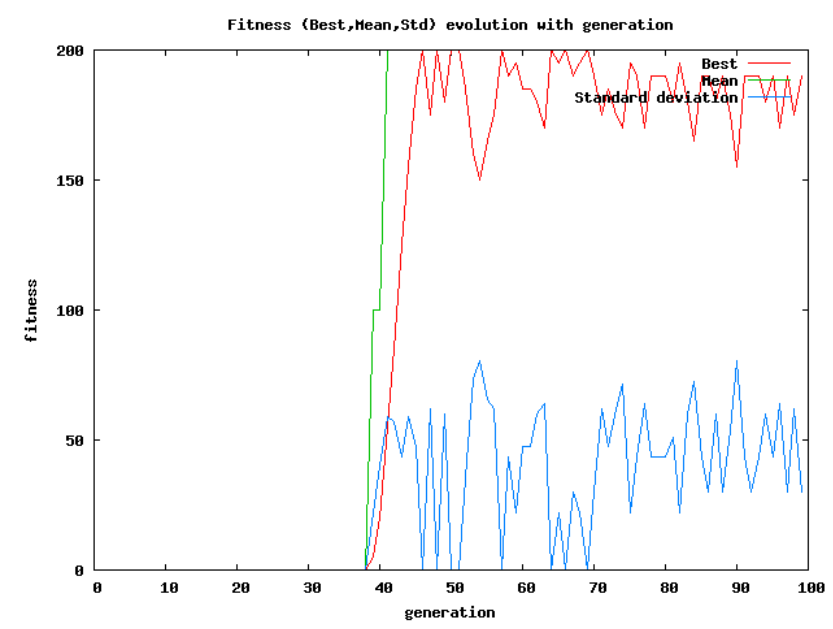

Fig. 9. Evolution of the best fitness; average finess and standard deviation of the best individual with generations

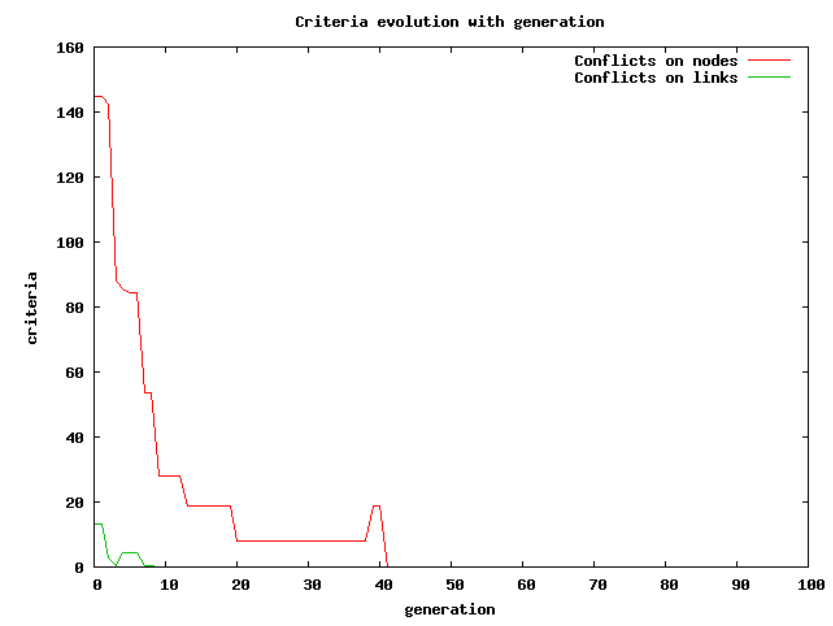

Fig. 10. Evolution of criteria $y_{1}, y_{2}$ with generations for the first scenario

rameters setting are not suitable anymore. We change in the following way in order to find optimal solutions :

\begin{tabular}{|c|c|}
\hline Pop size & 200 \\
Number of generation & 200 \\
Probability of Crossover & 0.3 \\
Probability of Mutation & 0.3 \\
\hline
\end{tabular}

The Figure 11 show the evolution of the fitness features with generations. An optimal solution is found after generation 60 with a fitness equal to 200 .

The associated evolution of criteria is given on

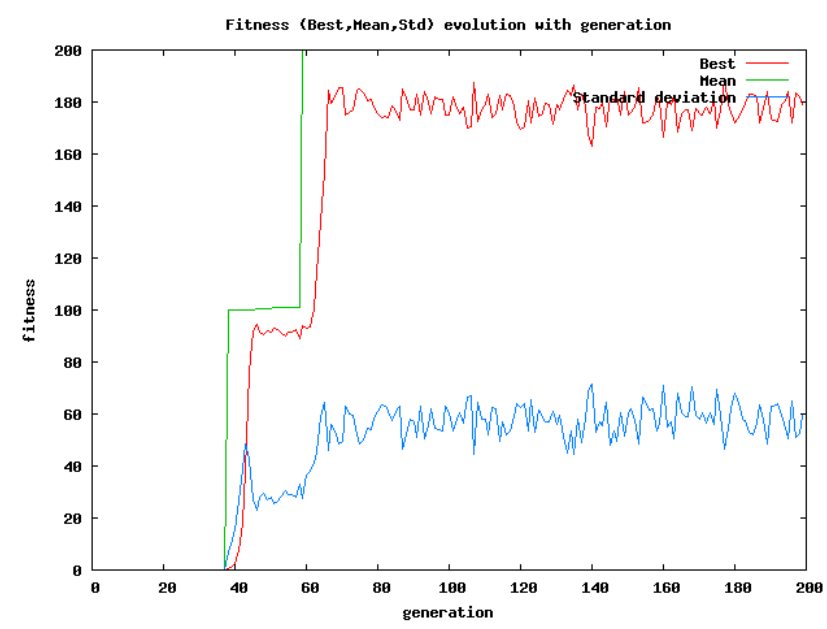

Fig. 11. Evolution of the best fitness; average finess and standard deviation of the best individual with generations

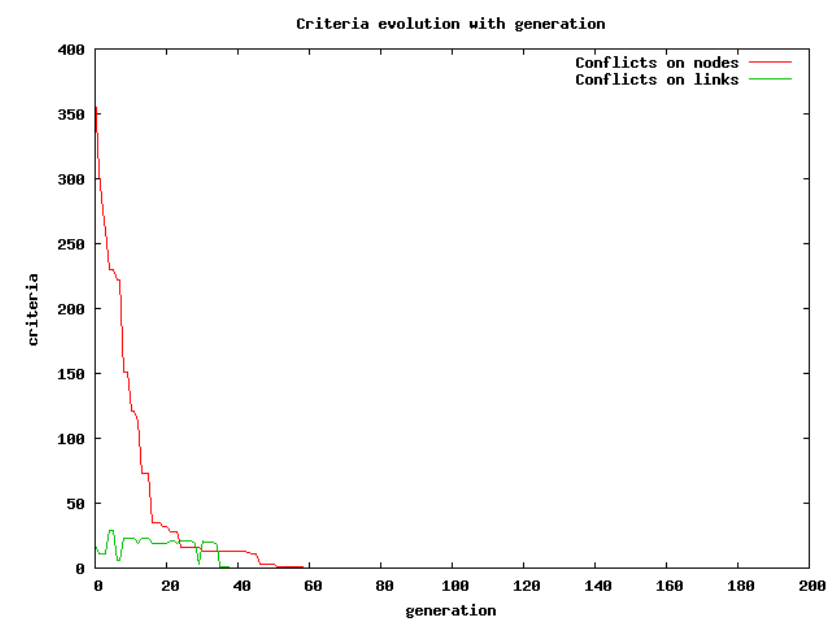

Fig. 12. Evolution of criteria with generations for the second scenario

Figure 12. As in the first experiment link conflicts diseppear first and node conflict are removed after generation 60 .

\section{Conclusion}

In this paper we have presented a new approach for the aircraft sequencing and merging problem in TMA. For each aircraft a new route may be selected in a discrete finite set and a new speed may be assigned.

A mathematical model has been developed for such problem with a description of the state space, constraints and objective function. A complexity analysis 
has shown that stochastic optimization is the most adapted approach to address such problem.

An Evolutionary Algorithm has been presented for which choromosome coding and operators have been developed.

Such algorithm has been applied to Canaria airport in Spain with real traffic demand samples. In both situation, all conflicts have been successfully removed on links and at merging points.

\section{Acknowledgment}

This research effort is partly funded by two main projects in the air traffic management domain with the aim of providing a significant contribution to the attainment of the common European goals set by the SESAR program and beyond. On is the CyCIT (TRA2008-05266/TAIR) by the Science and Innovation Ministry of the Spanish Government Discrete Event Simulation Platform to improve the flexible coordination of land/air side operations in the Terminal Manoeuvring Area (TMA) at a commercial airport; and the second project is The ATLANTIDA leaded by BR\&TE together with key research companies and Spanish universities, Universitat Autnoma de Barcelona (UAB) has been collaborating with Boeing Research and Technology Europe, ATOS-Origin and INDRA in the development of a causal model to improve $\mathrm{CD} / \mathrm{CR}$ algorithms performance.

\section{REFERENCES}

[1] H. Balakrishnan and B. Chandran. Scheduling aircraft landings under constrained position shifting. In The AIAA Guidance, Navigation and Control Conference, Keystone, Colorado., 2006.

[2] H. Balakrishnan and Bala G. Chandran. Algorithms for scheduling runway operations under constrained position shifting. Oper. Res., 58:1650-1665, November 2010.

[3] J.E. Beasley, M. Krishnamoorthy, Y. M. Sharaiha, and D. Abramson. Scheduling aircraft landings - the static case, 1998.

[4] T.A. Becker, D.R. Barker, and A.P. Smith. Methods for maintaining benefits for merging aircraft on terminal rnav routes. In Digital Avionics Systems Conference, 2004. DASC 04. The 23rd, volume 1, pages 2.E.1 - 21-13 Vol.1, 2004.

[5] L. Boursier, B. Favennec, E. Hoffman, A. Trzmiel, F. Vergne, and $\mathrm{K}$. Zeghal. Integrating aircraft flows in the terminal area with no radar vectoring. In 6th AIAA Aviation Technology, Integration and Operations Conference (ATIO) 25 - 27 September 2006, Wichita, Kansas, 2006.
[6] B. Chandran and H. Balakrishnan. A dynamic programming algorithm for robust runway scheduling. In American Control Conference, 2007. ACC '07, pages $1161-1166$, july 2007.

[7] A Cook, editor. European Air Traffic Management: Principles, Practice and Research. Ashgate Publishing Limited, 2007.

[8] N Durand and J.M Alliot. Ant colony optimization for air traffic conflict resolution. In 8th USA/Europe Air Traffic Management Research and Development Seminar, 2009.

[9] T. Farley, M. Kupfer, and H. Erzberger. Automated conflict resolution: A simulation evaluation under high demand including merging arrivals. In 7th AIAA Aviation Technology, Integration and Operations Conference (ATIO) $18-20$ September 2007, 2007.

[10] B. Favennec, T. Symmans, D. Houlihan, K. Vergne, and K. Zeghal. Point merge integration of arrival flows enabling extensive rnav application and cda - operational services and environment definition. Technical report, EUROCONTROL, 2008.

[11] E. Frazzoli, Z.H. Mao, J.H Oh, and E. Feron. Resolution of conflicts involving many aircraft via semidefinite programming. Technical report, MIT, 1999.

[12] D.E Goldberg. Genetic Algorithms in Search, Optimization and Machine Learning. Reading MA Addison Wesley, 1989.

[13] W.H Harman. Tcas: A system for preventing midair collisions. MIT Lincoln Laboratory Journal, Volume 2, Number 3, 1989., 1989.

[14] E. Hofhan, d. Ivanescu, C. Shaw, and K. Zeghal. Analysis of spacing guidance for sequencing aircraft on merging trajectorie. 2002.

[15] J. Hull, B. Barmore, and T. Abbott. Technology-enabled airborne spacing and merging. In Digital Avionics Systems Conference, 2004. DASC 04. The 23rd, volume 1, pages 2.B.4 - 2.1-9 Vol.1, oct. 2004.

[16] J.R Koza. Genetic Programming. MIT press, 1992.

[17] J Kurchar. A review of conflict detection and resolutionmodeling methods. Chemical Engineering Research and Design, 79(5):611 - 620, 2000. Fluid Flow.

[18] Boursier L., Favennec B., Hoffman E., Trzmiel A., Vergne F., and Zeghal K. Merging arrival flows without heading instructions. In 7th USA/Europe Air Traffic Management $R \& D$ Seminar, 2007.

[19] Z Michalewicz. Genetic algorithms + Data Structures $=$ Evolution Programs. Springer-verlag, 1992.

[20] M. Mondoloni, S.and Ballin and M. Palmer. Airborne conflict resolution for flow-restricted transition airspace. In AIAA's 3rd Annual Aviation Technology, Integration, and Operations (ATIO) Technical Forum, AIAA's2003, nov. 2003.

[21] L. Pallottino, E. Feron, and Bicchi A. Conflict resolution problems for air traffic management systems solved with mixed integer programming. Intelligent Transportation Systems, IEEE Transactions on, Vol. 3, No. 1. (2002), pp. 3-11., 2002.

[22] H.P Schwefel. Evolution and Optimum Seeking. Wiley, New York, 1995.

[23] A Vela. A mixed integer program for flight-level assignment and speed control for conflict resolution. In 48th IEEE 
Conference on Decision and Control and 28th Chinese Control Conference, 2009.

[24] D.A Vivona, R.A.and Karr. Pattern-based genetic algorithm for airborne conflict resolution. In American Institute of Aeronautics and Astronautics, Reston, Virginia, USA, oct. 2005. 